\title{
Rutile-anatase Composite Catalyst Formed by Coupling Anatase and Rutile Particles
}

\author{
A. Kalashnikova, N. Nikolenko*, J. Kalashnikov, A. Kostynyuk \\ Faculty of Silicate Technology, Ukrainian State University of the Chemical Engineering, Dniepropetrovsk, 49005, Ukraine \\ *Corresponding Author: n_nikolenko@ukr.net
}

Copyright $(2013$ Horizon Research Publishing All rights reserved.

\begin{abstract}
Anatase-type $\mathrm{TiO}_{2}$ particles were formed on a surface of rutile particles by thermal crystallization from amorphous titan oxyhydroxide that was obtained from the aqueous solution of $\mathrm{TiOCl}_{2}$ using urea hydrolysis. Special attention was paid to obtain rutile-anatase composite with reproductive physico-chemical properties by control of synthesis parameters. Catalytic activity of the synthesized composite was studied on photoinduced oxidation of formaldehyde vapours in a flow reactor.
\end{abstract}

Keywords Titanium Dioxide, Rutile-anatase Composite, Formaldehyde, Photocatalytic Oxidation Process

\section{Introduction}

The investigations of catalytic processes induced by light on semiconductors' surface have drawn attention of many researchers because of promising perspectives of catalytic technology [1]. Both fundamental and applied researches have the common problem of choosing optimum semiconducting material. The main task is to find the material which is the most active and has the highest degree of light energy use [2].

At present, titanium dioxide is considered to be one of the best photocatalysts which is industrial product as well as product of synthesis in many research laboratories. It is also well known, that anatase form of titanium dioxide demonstrates the highest photocatalytic activity. The cause of this phenomenon remains unclear because of a number of exceptions. For example, one of the best industrial photocatalysts - titanium dioxide Degussa P-25 - includes $20 \%$ of rutile and $80 \%$ of anatase modifications. A number of authors explain high photocatalytic activity of rutile-anatase structure by phase boundary, which favors to increase concentration of electron-hole charge carrier by inhibition of the process of their recombination [3].

In our opinion, studies on influence of phase boundary in titanium dioxide on its photocatalytic properties can be carried out by means of rutile-anatase composites. The question is not mechanical mixture of anatase and rutile particles, but such a structure when on the surface of relatively large particles of rutile (or anatase) microparticles of titanium dioxide with rutile (or anatase) crystal structure are formed. During synthesis of this composite it is possible to control effectively relation of two crystalline phases just on particle surface, i.e. where photocatalytic redox process of destruction and oxidation of organic compounds take place.

The present work is aimed at developing rutile-anatase composite process by titanium(IV) oxyhydroxide precipitation on the surface of rutile particles at controlled degree of mother liquor supersaturation with its subsequent dehydration and calcination to form crystalline phase of anatase. To obtain composites with reproductive physico-chemical properties during synthesizing special attention was paid to control of its basic parameters. Concentrations of metal ions, hydrogen and rutile particles, the degree of mother liquor supersaturation, the time of hydroxide ageing, temperature and time of composite drying and calcination were considered as above mentioned parameters.

By way of working hypothesis for producing highly active photocatalyst we consider the task of obtaining the composite in which some part of rutile particles surface would remain free of anatase microparticles. The point is that light extinction coefficient of rutile in the near-ultra-violet region is higher than that of anatase. This is explained by the fact that the width of rutile forbidden band is smaller than anatase. Therefore, light absorption band by rutile surface is partially shifted from UV-region into visible spectrum. It was assumed that absorption of light quantum by rutile would favour increasing concentration of free charge carrier not only in rutile structure, but in anatase "superstructure" of composite particle. A similar hypothesis about the possibility of enhancing visible-light-induced photocatalytic activity by means of the composites of different catalysts based on the coupling suitable wide and narrow band-gap semiconductors also have been expressed authors [4].

In this paper the synthesis of rutile-anatase composite catalyst with $10 \%$ content of anatase is described. Catalytic properties of the substance are investigated by an example 
of photoinduced oxidation of formaldehyde vapours in model flow reactor.

\section{Materials and Methods}

Modification of rutile surface by anatase particles was carried out by precipitation of titanium(IV) oxyhydroxide in presence of rutile suspension. To $5.00 \mathrm{~g}$ of rutile sample were added $100 \mathrm{ml} 1 \mathrm{M}$ solution of acid $\left(\mathrm{HCl}\right.$ or $\left.\mathrm{H}_{2} \mathrm{SO}_{4}\right)$ and 5.50 $\mathrm{ml} 1.140 \mathrm{M}$ solution of $\mathrm{TiOCl}_{2}$. Precipitation by aqueous ammonia was conducted at room temperature, precipitation by urea was carried out by heating up to $70^{\circ} \mathrm{C}$. Conditions of synthesis, washing, drying and calcination of the precipitate are described in the article.

Synthesis of rutile as a composite substrate was carried out by calcination of titanium(IV) oxyhydroxide at $900^{\circ} \mathrm{C}$ for 10 hours. Titanium(IV) oxyhydroxide have been prepared by mixing $\mathrm{TiOCl}_{2}$ and $\mathrm{NH}_{4} \mathrm{OH}$ solutions at $298 \mathrm{~K}$ and $\mathrm{pH}=11,0$ in a reactor with stirrer. Before calcination precipitate of $\mathrm{TiO}(\mathrm{OH})_{2}$ was filtered and extensive washed with deionized water, isopropyl and amyl alcohols. The particle size distributions were determined by sedimentation analysis. A single-mode distribution centered at $13 \mu \mathrm{m}$ was found which leads to an average equivalent radius of rutile particles of $21 \pm 2 \mu \mathrm{m}$.

Methodology of experiments on photocatalytic oxidation of formaldehyde has been described in [5]. The study was performed in a model flow photoreactor. Schematic representation of used reactor is shown in fig. 1.

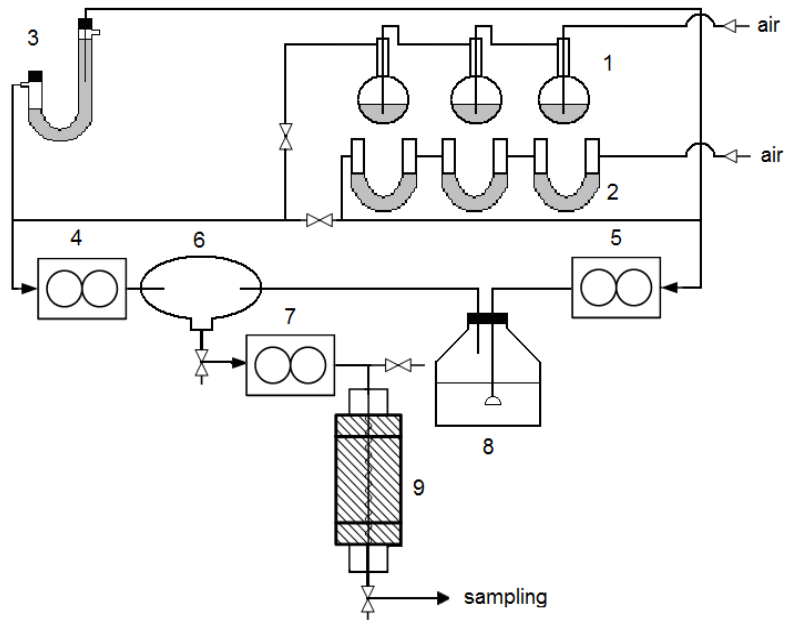

Figure 1. Scheme of facility for study of photocatalyc oxidation of formaldehyde. 1-flasks with sulfuric acid; 2- containers with granulated activated carbon and $\mathrm{CaCl}_{2}$; 3- control vessel; 4,5,7- flow meters; 6-gas mixing flask; 8-evaporator; 9-flow photoreactor.

The morphologies and microstructures characterizations were performed on a Selmi scanning electron microscope (SEM). The crystalline phase composition was analysed by X-ray diffraction (XRD) using a X'Pert Powder instrument fitted with a hemispherical analyzer with $\mathrm{CuK} \alpha$ radiation. Thermal studies (DTA-DSC, TGA) were performed on
SDT Q600 thermal analyzer.

\section{Results and Discussion}

A special attention in modification of rutile surface by anatase particles and obtaining of the rutile-anatase composite with reproducible properties was paid to control of key-parameters of all stages of synthesis process. This process was regarded as sequence of the following stages: a) preparation of rutile suspension in titanium(IV) salts solutions; b) precipitation of titanium oxyhydroxide at controlled changes of mother liquor supersaturation; c) ageing of titanium oxyhydroxide in mother liquor; d) washing, drying and calcination of obtained composite.

\subsection{Standardization of Initial Parameters of Synthesis Process}

It is known for certain that ions of titanium(IV) in aqueous solutions are subjected to hydrolysis and polymerization. In diluted chloride solutions at $\mathrm{C}(\mathrm{H}+)>0.1 \mathrm{M}$ titanium ions are mainly in the form of $\mathrm{TiO}^{2+}[6]$. With the increase of concentration polynuclear cationic complexes are formed. According to the authors [7] in $0.02 \mathrm{M}$ titanium(IV) solutions complexes with the composition $\left[(\mathrm{TiO})_{8}(\mathrm{OH})_{12}\right]^{+4}$ become predominant form. On account of Coulomb repulsion the union of $\mathrm{TiO}^{2+}$ or $\mathrm{TiO}(\mathrm{OH})^{+}$cations into polynuclear complex appears to be unlikely. Therefore, hydroxo-complex polymerization is usually regarded as successive attachment of uncharged (neutral) particles to charged complex particles, for example: $\left[\mathrm{TiO}\left(\mathrm{OH}_{2}\right)\right]^{0}$ or $\left[(\mathrm{TiO})_{\mathrm{x}}(\mathrm{OH})_{2 \mathrm{x}}\right]^{0}$, where $\mathrm{x}$ is polymerization degree. Apparently, due to low concentration of neutral complexes in strong acidic solutions the rate of establishing equilibrium for the process involved is rather low. In chemistry of complex compounds low rate of ligand-exchange reactions for ions of titanium(IV) is explained by their low lability. Large positive charge of cation and its relatively small radius are considered to increase activation energy of replacement reaction which results in decreasing rate of intraspheric ligand exchange into hydroxide ions or water molecules from solution environment.

Thus, for standardization of initial composition of titanium(IV) hydroxo-complexes after dilution or change of solution acidity it is necessary to wait for hydrolytic equilibrium to be completed. Otherwise, hydroxide deposition will proceed at uncontrolled composition of titanium complexes; this can influence the parameters of solid phase nucleus formation.

Another no less important parameters of composite synthesis, to our opinion, appears to be adsorption of titanium hydroxo-complexes on rutile surface. It is known, that multicharged ions are able to be specifically absorbed on oxide surfaces. Such adsorption can be regarded as the process of surface hydrolysis when a part of coordination sphere ligands are replaced by $\mathrm{OH}$-groups on oxide surface. 
According to that there must not be any principal differences for auto adsorption $\left[(\mathrm{TiO})_{\mathrm{x}}(\mathrm{OH})_{2 \mathrm{x}}\right]^{\mathrm{z}}$ on rutile surface. Surface of formed hydroxo-complexes can be considered as crystallization centre of titanium hydroxide phase. Apparently, at various content of such crystallization centre on the surface of rutile particles composites with different distribution of hydroxide particles both according to their number and size can be formed. That is why for standardization of initial conditions for composite synthesis in the solution of rutile powder suspension and titanium salt, adsorption equilibrium should be complete.

Invariability of solution acidity may serve as common sign of attaining hydrolytic and adsorption equilibrium. To confirm this conclusion experimentally we carried out studies on dynamics of acidity change in $1 \mathrm{M}$ solution of hydrochloric acid in quantity of $100 \mathrm{ml}$ when $5 \mathrm{~g}$ of rutile powder and $5.5 \mathrm{ml}$ of $1.14 \mathrm{M} \mathrm{TiOCl}_{2}$ solution with initial $\mathrm{pH}=0$ was added. It was found that after quick increase of $\mathrm{pH}$ (by 0.4 unit) caused by hydrolytic processes proceeding on titanium oxide surface, during the following 5 hours $\mathrm{pH}$ of the solution increases by 0.28 unit more. This can be explained by slow rate of hydrolysis process and adsorption of titanium(IV) cation complexes.

Therefore, to standardize initial parameters of synthesis solution of titanium(IV) salt after dilution, change of acidity and sample addition $\mathrm{TiO}_{2}$ should be preliminary kept for no less than 5 hours for completing hydrolytic and adsorption equilibrium.

\subsection{Standardization of Titanium Oxyhydroxide Precipitation and Ageing Parameters}

It is well known that appearance of a new phase is possible in metastable system which is disturbed from the equilibrium as a result of changes in chemical composition (oversaturation method) or physical action (e.g., temperature and/or pressure in hydrothermal method of precipitation). The formation of a new phase begins with the appearance of so-called critical nuclei. It is known that if there is seeding agents the probability of heterogenous nucleation as contrasted to by homogeneous nucleation is considerably increased. In such cases the crystallization goes on a surface of seeding agents.

On regularities of heterogenous nucleation we have developed method for obtaining rutile-anatase composite by titanium(IV) oxyhydroxide precipitation in presence of rutile suspension under conditions of controlled change of mother liquor acidity [8]. In result of preliminary test it was found that $\mathrm{TiO}(\mathrm{OH})_{2}$ precipitation by ammonia hidroxide from titanium(IV) hydrochloride solutions at $\mathrm{pH} \sim 2$ lead to formation of a sol of titanium(IV) oxyhydroxide. made that The reason of sol formation in this process is homogenous precipitation when nuclei of oxyhydroxide particles are formed not on the rutile particles surface in solution volume. During nuclei growth because of high charge and small mass they remain in the solution forming stable sol.

According to classical theory of precipitation formation, in order to retard nucleation in solution volume it is necessary to decrease rate of precipitant agent addition and thus to lower degree of mother liquor supersaturation. As stated above, heterogenous nucleation is in fact of process of polymolecular adsorption of uncharged hydroxo-complexes since only these particles don't suffer considerable electrostatic repulsion when approaching charged surface of oxide. Taking into account relatively low rate of titanium ions hydrolysis we conclude that the lower the rate of precipitator addition, the higher the degree of fit ions transformation into neutral form $\left[(\mathrm{TiO})_{\mathrm{x}}(\mathrm{OH})_{2 \mathrm{x}}\right]^{0}$ and the more likely their adsorption on rutile surface.

Decrease of hydroxide precipitation rate was realized by 2 methods: by gradual (stepping) addition of precipitator and by method of arising reagents. In the first case ammonia solution was added in suspension solution by small portions in such a way that the value of solution $\mathrm{pH}$ changed by no more than 0.1 unit during 5 hours (the time for the equilibrium of titanium ions hydrolysis to be completed). The change in acidity was done within the interval from 0.6 (beginning of hydroxide precipitate formation) to 2.0 units.

Urea was added in the titanium(IV) solution for realization of arising reactants way. As it's known, during urea hydrolysis concentration of hydrogen ions in a solution gradually decreases:

$$
\left(\mathrm{NH}_{2}\right)_{2} \mathrm{CO}+2 \mathrm{H}_{2} \mathrm{O}+\mathrm{H}^{+} \rightarrow \mathrm{HCO}_{3}^{-}+2 \mathrm{NH}^{4+} \text {. }
$$

It slowly displaces equilibrium of hydrolysis of metals cations, owing to particles of a hydroxide solid phase are formed at low supersaturation degrees. In both cases precipitation carried out from sulfate solutions that allowed to prevent probable precipitation of titanium hydroxide in the rutile form. As is known, precipitation of titanium hydroxide from sulfate solutions leads to the formation of anatase form irrespective of $\mathrm{pH}$ liquor solution.

As a result of the performed experiments it was found that when precipitating fit hydroxide by method of gradual addition of a precipitator a stable sol in quantity $\sim 75 \%$ of total hydroxide precipitator mass is formed in suspension solution. Thus, even when the rate of precipitator addition decreases by 1000 times a considerable part of titanium hydroxide is formed not on the surface of rutile particles but in the volume of the solution. In our opinion, obtained results can be explained by the fact that in contrast to mechanism of ion crystal nuclei formation, heterogeneous and homogeneous formation of titanium hydroxide and other d-metals follows the same mechanism. The essence of the latter is the growth of polymeric chain of polynuclear hydroxo-complexes. That is why probability of heterogeneous and homogenous precipitation of hydroxides is the same and therefore the processes proceed at practically equal rate.

It should be noted, that independently of the precipitator addition rate in reaction zone (i.e. on the boundary of ammonia solution drop contact with the titanium salt solution) high degree of solution supersaturation takes place. For example, in studied experimental conditions concentration of $\mathrm{OH}$ - ions changes from 2 to $10-12 \mathrm{~mol} / \mathrm{l}$ 
and of $\mathrm{TiO}^{2+}$ from 0.06 to $10-5 \mathrm{~mol} / \mathrm{l}$. Therefore, hydroxide precipitation proceeds very quickly and the relation of hydroxide amounts precipitated heterogeneously and homogenously is determined by the volume of reaction zone and rutile particles content in it rather than by precipitation rate. Precipitating hydroxide by method of appearing reactants shows that the change of supersaturation degree in reaction zone will be considerably smaller. For example, when using urea hydrolysis concentration of hydroxide-ions (at $\mathrm{pH}$ change of mother liquor from 0 to 2 units) changes only by 100 times. This decrease in the degree of mother liquor precipitation results in abrupt decreasing of hydroxide precipitation rate, and, therefore, should cause formation of monodisperse particles. This conclusion was verified by experiment by the authors [9], who had determined that at fit hydroxide precipitation, almost spherical particles with the size of $\sim 2 \mu$ were formed by aggregating of primary anatase nanoparticles (size $4-5 \mathrm{~nm}$ ). The authors [10] found that up to 500 -fold excess of urea the hydroxide precipitation proceeds predominantly in heterogeneous manner (for the experiments plates of glass, polyethylene and polypropylene were employed).

It was also found that at further rise of urea content homogenous precipitation predominated. Besides, fit hydroxide precipitates in a form of anatase and only at decrease of urea excess by less than 150 times precipitate of titanium hydroxide may be formed as the mixture of both crystal structures - rutile and anatase ones.

Therefore, in the experiments carried out in our studies, 250 -fold excess of urea in ratio to initial content of titanium was used. Urea hydrolysis was initiated by heating solutions up to $70^{\circ} \mathrm{C}$. Precipitation was performed at continuous stirring of the suspension. The process was caused when mother liquor acidity reached $\mathrm{pH}=2.0$. It was assumed that in the case when coagulation-stable sol of hydroxide is formed, the sol can be easily separated from the composite precipitate by washing or filtering.

The results of the experiments have shown, that at titanium hydroxide precipitation by urea, $\mathrm{TiO}(\mathrm{OH})_{2}$ sol is not practically formed. Under the given experimental conditions urea is likely to decompose on rutile particles surfaces thus giving rise to alkalifying solution at oxide particles surface and initiating polymerization of titanium hydroxo-complexes $\left[(\mathrm{TiO})_{x}(\mathrm{OH})_{2 \times}\right]^{0}$. Since the rate of polymerization is much higher than diffusion rate of polynuclear hydroxo-complexes being formed in the solution volume, greater part of critical nuclei of fit hydroxide remains bound to rutile surface.

To confirm the possibility of catalytic action of rutile on urea hydrolysis we compared the rate of acidity change of urea solution at constant temperature both in presence and absence of $\mathrm{TiO}_{2}$ suspension (Fig.2).

It was determined, that in such equal conditions (i.e. initial acidity of solutions, urea concentration, temperature, heating rate and rate of solution stirring) in presence of rutile the rate of urea hydrolysis is noticeably higher. Was assumed that urea is adsorbed on $\mathrm{TiO}_{2}$ surface, for example, by H-bonds between its $\mathrm{NH}_{2}$-groups and hydroxyl groups of titanium oxide. As a result of adsorption the strength of bonds between nitrogen atoms and those of carbon in $\mathrm{CO}\left(\mathrm{NH}_{2}\right)_{2}$ molecule can decrease thus diminishing activation energy of hydrolysis. Therefore, possibility of rutile-anatase composite synthesis by the method combining processes of heterogeneous nucleation and gradual accumulation of the precipitator is determined by the ability of the reagent chosen to decompose just in the reaction zone (i.e. on the surface of rutile microparticles) rather than by the precipitation rate and the degree of supersaturation in the reaction zone.

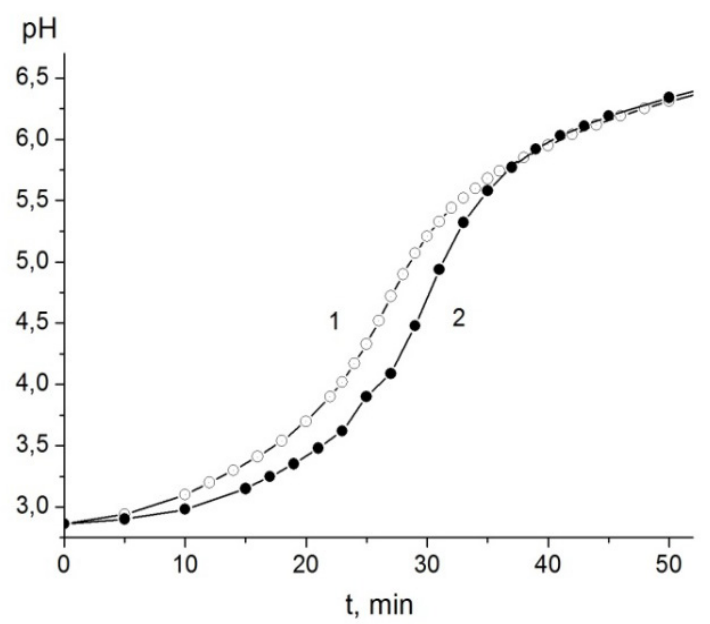

Figure 2. Change of $\mathrm{pH}$ solutions of urea after their heating up to $70^{\circ} \mathrm{C}$ in absence (1) and at presence (2) rutile (urea concentration - $133 \mathrm{mg} / \mathrm{ml}$, weight of rutile powder $-5 \mathrm{~g}$, solution volume $-150 \mathrm{ml}$ )

To determine the time for polymerization of titanium oxyhydroxide precipitate to be completed we have studied the dynamics of mother liquor acidity changes. In Fig.3 typical kinetic curves of solution acidity change over the precipitate of fresh-precipitated titanium hydroxide are shown.

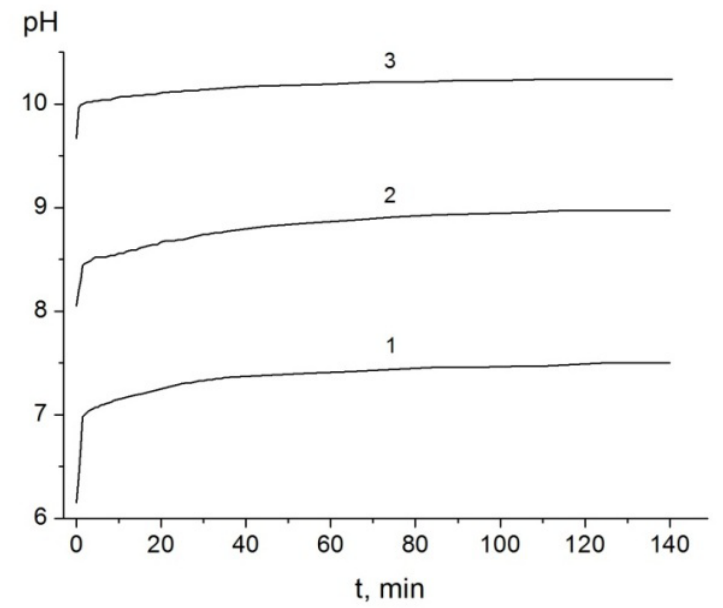

Figure 3. Change of solution $\mathrm{pH}$ with precipitate of titanium oxyhydroxide (1- $6.15 ; 2-8.05 ; 3-9.67)$

It was found that after addition of $\mathrm{NH}_{4} \mathrm{OH}$ solution the acidity of suspension continues to decrease quickly and only 
in $\sim 2.5 \mathrm{~min}$ the rate of its change diminishes rapidly. Then, on kinetic curves $\mathrm{pH}$ exponentially increases during $\sim 120$ min. In our opinion, the first stage of solution acidity change can be explained by slow equalization of precipitator concentration in the solution volume, since oxyhydroxide particles are quickly formed on the boundary of $\mathrm{TiOCl}_{2}$ and $\mathrm{NH}_{4} \mathrm{OH}$ solutions contact. Exponential growth of $\mathrm{pH}$ during next 2 hours is consistently explained by ions $\mathrm{OH}^{-}$releasing with the formation of intraparticle olative (bridged) bonds between neighboring titanium cations 1 :

$$
2 \equiv \mathrm{Ti}-\mathrm{OH} \rightarrow \equiv \mathrm{Ti}(\mathrm{OH}) \mathrm{Ti} \equiv+\mathrm{OH}^{-} .
$$

Thus, investigation of mother liquors acidity at ageing of titanium hydroxide has shown that in 2 hours the main fraction of $\mathrm{OH}$-groups accessible for interaction is transformed into olative bonds. We can expect that hydroxide particle aggregates acquire extra rigidity and with further intraparticle polymerization of gel they can keep their structure.

\subsection{Standardizing Parameters of Rutile-Anatase Composite Washing, Drying and Calcinations.}

Precipitating rutile-anatase composite from mother liquor without breaking hydroxide "superstructure" on the surface of rutile particles is rather complex task. Suspension filtering or centrifuging leads to packing of obtained deposits. This tends to particles aggregation as a result of initiated processes of olation and oxalation. As a result of hydroxide precipitate washing by water in accordance with the acid-salt concentration ratio, in mother liquor the processes of the precipitate coagulative aggregation and peptization can be initiated. Therefore, we used $0.01 \mathrm{M}$ solution of hydrochloric acid as washing liquid. This permitted to remove ions of ammonium sulfate from the solution and to maintain the charge on hydroxide surface constant. Chloride-ions were removed by washing the precipitate with organic solvent. At washing decantation method was used: solution over the precipitate was drawn off with siphon, then a small amount of $0.01 \mathrm{M}$ solution of hydrochloric acid was added, the suspension was stirred and the operation was repeated up to the complete suspension washing off ammonium sulfate impurities. Similar method was used for washing the precipitate by polar organic solvents - isopropyl and amyl alcohols. Using isopropanol allows to extract quickly a great amount of water from the precipitate as well as to wash precipitator from chloride-ion impurity. The subsequent substitution of isopropanol with pentanol was done to prevent hydroxide particles aggregation at its drying because the high boiling temperature of amyl alcohol makes water azeotropic distillation possible [11].

To choose the conditions for oxide-hydroxide composite

\footnotetext{
${ }^{1}$ The subsequent deprotonation of bridging $\mathrm{OH}$-ligands is possible with the absorption of protons by $\equiv \mathrm{TiO}-$ and $\equiv \mathrm{TiOH}$ groups:

$\equiv \mathrm{Ti}(\mathrm{OH}) \mathrm{Ti} \equiv \rightarrow \equiv \mathrm{TiOTi} \equiv+\mathrm{H}^{+}$.

$\equiv \mathrm{TiOH}+\mathrm{H}^{+} \rightarrow \equiv \mathrm{TiOH}_{2}^{+}$.

$\equiv \mathrm{TiO}^{-}+\mathrm{H}^{+} \rightarrow \equiv \mathrm{TiOH}$
}

transformation into its oxide rutile-anatase form DTA-DSC and XRD studies were performed. It was found that complete dehydration of oxide-hydroxide composite occurs at temperature $180-200^{\circ} \mathrm{C}$ (DTA curve shows relatively high endothermal peak). Such high temperature is explained by removing coordination-bound water and dehydroxylation of titanium hydroxide. At further heating we can observe small exothermal peak at $410-430^{\circ} \mathrm{C}$ which is evidence of X-ray amorphous oxyhydroxide transformation into anatase crystalline form.

Thus, drying was performed in two steps keeping composite precipitate for one hour at temperature $135^{\circ} \mathrm{C}$ (boiling in amyl alcohol for removing coordination-bound water) and at temperature $200^{\circ} \mathrm{C}$ (to remove alcohol traces and to get complete dehydration of the composite). In order to increase titanium oxyhydroxide degree of crystallinity the samples of composite was calcinated at 300, 400, 600 and $800^{\circ} \mathrm{C}$ for 3 hours. It should be noted, that together with oxyhydroxide dehydration the nature of its bond with rutile surface as bridges $\equiv \mathrm{Ti}(\mathrm{OH}) \mathrm{Ti} \equiv$ as well as H-bonds between $\mathrm{OH}$-groups in the point of anatase and rutile particles contact are able to dehydrate and to be transformed into stronger oxo-bonds. At further composite calcinations structural change of particles "contact points" takes place, the former leads to ordering and increasing the number of oxo-bonds between rutile phase and that of anatase.

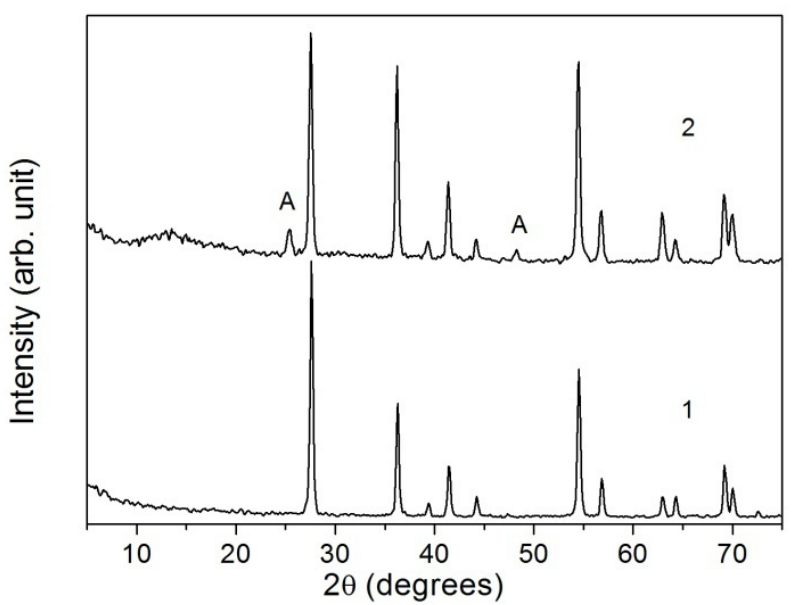

Figure 4. XRD profiles for rutile-substrate (1) and rutile-anatase composite (2) after calcination at $600^{\circ} \mathrm{C}$ for 3 hours. (A - anatase diffraction peak).

The crystalline phase of synthesized composite was analysed by XRD. The starting substrate of composite shows only diffraction peaks of rutile (Fig.4, curve 1). The XRD diagram of rutile-anatase composite shows changes of particle size and phase composition during annealing. Up to $400^{\circ} \mathrm{C}$ we observed a relatively large content of amorphous titanium oxide and indistinctive narrowing of anatase diffraction peaks at 25.30 and 48.30 corresponding to the particle growth of this phase. It should be noted that the presence of the amorphous band in the X-ray spectrum may be due to the very small particle size of the anatase powder precipitated [8]. Significant peak narrowing and formation of 
anatase phase took place between 400 and $600^{\circ} \mathrm{C}$ (Fig.4, curve 2).

Electron microscopy studies of obtained composites did not allow us to identify an individual phase of anatase at rutile surface. Therefore, for identification of particles "superstructure" the sedimentation of the titanium hydroxide on the surface of rutile particles was carried with at presence of zirconium(IV) ions which was used as a probe.

Coprecipitation of titanium and zirconium hydroxides, was carried from a solution containing $0.6 \mathrm{M} \mathrm{TiOCl}_{2}$ and 0.1

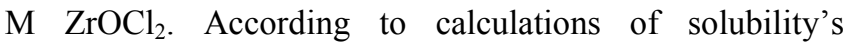
appropriate hydroxides, at such concentrations precipitation $\mathrm{TiO}(\mathrm{OH})_{2}$ begins at $\mathrm{pH} 0.6$, and $\mathrm{Zr}(\mathrm{OH})_{4}$ - at $\mathrm{pH}$ 1.9. Since the deposition was performed at $\mathrm{pH} 2.5$, the precipitate contained a mixture of the both hydroxides.

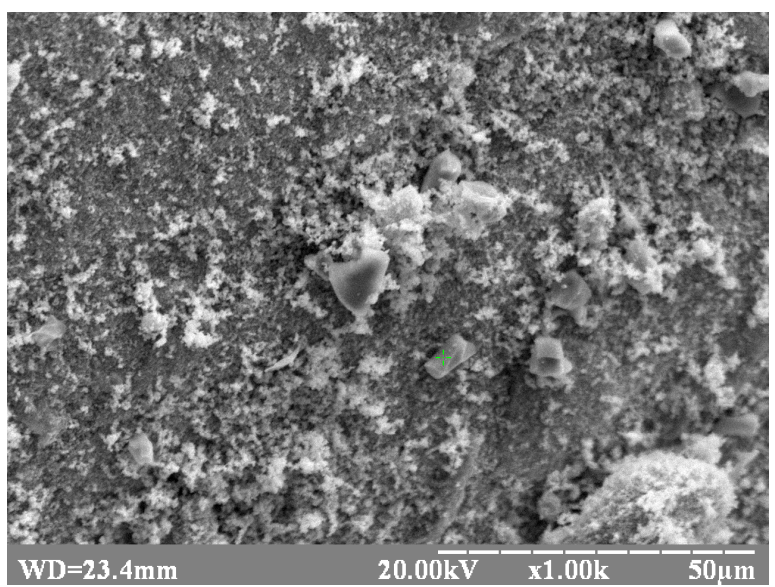

Figure 5. The SEM image of the rutile-anatase composite sample.

The SEM-analysis of the dark sites of surface showed presence of titanium and absence of zirconium atoms, which allows to consider the dark areas of the composite surface to rutile phase. Determination of structure carried out for microparticles aggregates which located on the rutile surface (light sites of a picture) showed presence of titanium atoms and zirconium atoms in the ratio 1,7:1. Such structure of particles is explained by coprecipitation of titanium and zirconium ions.

Analysis of Fig.5 shows that at the rutile surface is randomly located anatase particles with similar size $(\sim 1$ $\mathrm{mkm})$ and shapes. It should be noted that according to the authors [8] precipitation of titanium oxyhydroxide at $\mathrm{pH}=2$ are formed phase with primary crystallite size less than $0.1 \mu$. Apparently, under the chosen conditions of synthesis primary particles of titanium oxyhydroxide are aggregated in larger units as a result of ollation and oxollation processes.

\subsection{Studies of Photocatalytic Properties of Rutile-Anatase Composite}

Photocatalytic activity of synthesized rutile-anatase composite was studied on photoinduced oxidation of formaldehyde vapours in a flow reactor. Formaldehyde is known to be a widespread organic air pollutant with relatively low $\left(0.5 \mathrm{mg} / \mathrm{m}^{3}\right)$ maximum permissible concentration. At present investigations into photocatalytic oxidation of formaldehyde were carried out by many authors [12-14]. It was shown the possibility of rapid photoinduced oxidation of formaldehyde on titanium dioxide catalysts with two steps: $\mathrm{HCHO}$ is adsorbed on the hydroxyl groups on the $\mathrm{TiO}_{2}$ surface via hydrogen bonding and rapidly converts to the formiate species, then formiate intermediates is converted into final product of $\mathrm{CO}_{2}$ and $\mathrm{H}_{2} \mathrm{O}$ [5,15-18].

In this study investigations of photocatalytic properties of synthesized composite and a number of titanium dioxides in amorphous and crystalline forms were carried out in a model flow photoreactor. Preliminary experiments have proved that in absence of UV-radiation or catalyst concentration of formaldehyde vapours at reactor's inlet and outlet remains unchanged for the whole range of loading. Determination of gas mixture composition in presence of UV-radiation and catalyst has shown that with the decrease of reaction mixture flow rate through the reactor concentration of formaldehyde reduces gradually, $\mathrm{CO}_{2}$ concentration gradually increases and the amount of formic acid first slightly increases and then slowly reduces. The example of graphic representation of relationship between concentration of the determined components of the reaction mixture and residence time ${ }^{2}(\Delta \tau$ $=\tau-\tau_{0}$ ) of photocatalytic oxidation reaction is shown in Fig.6. Dots show the experimental data at $\mathrm{T}=293 \mathrm{~K}$ and the curves are plotted on the basis of two-steps mathematical model of photoinduced oxidation of formaldehyde in approximation of pseudo-first-order rate reaction [5]. Accordingly to this model, observed rate constants for data shown in Fig. 6 are equal to $2.13 .10^{-5} \mathrm{~s}^{-1}$ per $1 \mathrm{~cm}^{2}$ of irradiated reactor surface for the first stage $(\mathrm{HCHO} \rightarrow \mathrm{HCOOH})$ and $2.55 .10^{-5} \mathrm{~s}^{-1}$ for the second stage $\left(\mathrm{HCOOH} \rightarrow \mathrm{CO}_{2}\right.$ ) of oxidation process.

It is necessary to note that the results of theoretical calculations agree with the experimental data well provided the greater amount of formic acid is on the catalyst surface but not in gas phase. In Fig.6 curve 3 depicts the result of calculations for the amount of formic acid in the reactor gas phase with due account of its adsorption (shown by the curve 2 ). This result is in accord with the data of the authors $[16,17]$ who found that when formaldehyde undergoes oxidation on $\mathrm{TiO}_{2}$ catalyst the formiates which are collected on the oxide surface are being quickly formed.

2 Residence time of the reaction was calculated as relationship between the volume of reactor's reaction zone and the flow rate of the substances through the system. The value of reference time $\tau_{\mathrm{o}}$ at which the concentration of formaldehyde in the reactor accounted for its initial concentration measured in no UV radiation condition was taken as a starting point. 


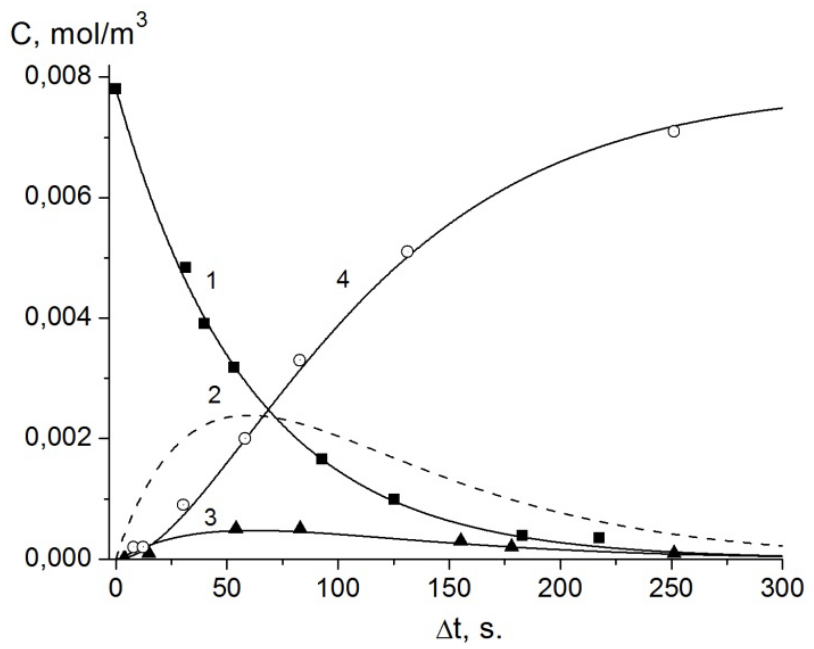

Figure 6. Dependence of formaldehyde concentration (1), formic acid $(2,3)$ and carbon dioxide (4) on residence reaction time of photooxidation at rutile-anatase composite calcinated at $600^{\circ} \mathrm{C}$ for 3 hours. Curve (2) depicts the calculation results of adsorbed formic acid.

The content of formaldehyde in a gas stream at different temperatures was determined for definition of limiting stage of process rate. It was found that in a temperature range $293 \div 323 \mathrm{~K}$ oxidation process proceeds in a kinetic mode. For all examined oxides the obtained rate constants of photoinduced oxidation of formaldehyde are well linearized on the coordinates of Arrhenius equation in this temperature range with the apparent activation energy $6 \div 25 \mathrm{~kJ} / \mathrm{mol}$. In Fig. 6 the dependences between apparent activation energies of photoinduced oxidation of formaldehyde to formic acid and calcination temperatures of titanium dioxide and composite $(10 \%$ anatase $+90 \%$ rutile) are shown.

According to data of Fig.7, crystalline structures of titanium dioxide play an important role in the formaldehyde photoinduced oxidation rate. The oxidation of formaldehyde with the minimum activation energy is achieved at usage as catalyst a crystalline anatase obtained by calcination of titanium oxyhydroxide in temperature range $400-600^{\circ} \mathrm{C}$ (curve 1). At the same time, the partial oxidation of formaldehyde on rutile-anatase composite with 10-w.t.\% content of crystalline anatase also is realized with the minimum activation energy equal to $\sim 6 \mathrm{~kJ} / \mathrm{mol}$ (the curve 2 ). So far as the $10 \%$ excess of crystalline anatase particles is deficient for complete covering of rutile surface that it is possible to draw a conclusion about synergism of photoinduced catalytic activity of two coupled crystalline structures of titanium dioxide. Apparently, that phase boundary of anatase and rutile promotes formation of new adsorptive centers on a surface of a composite material and

favors the increase of concentration of photo-generated electrons and holes due to decrease of their recombination rate. Our experimental result implies that the mixed phase of anatase and rutile can markedly enhance the photocatalytic activity of titanium dioxide.

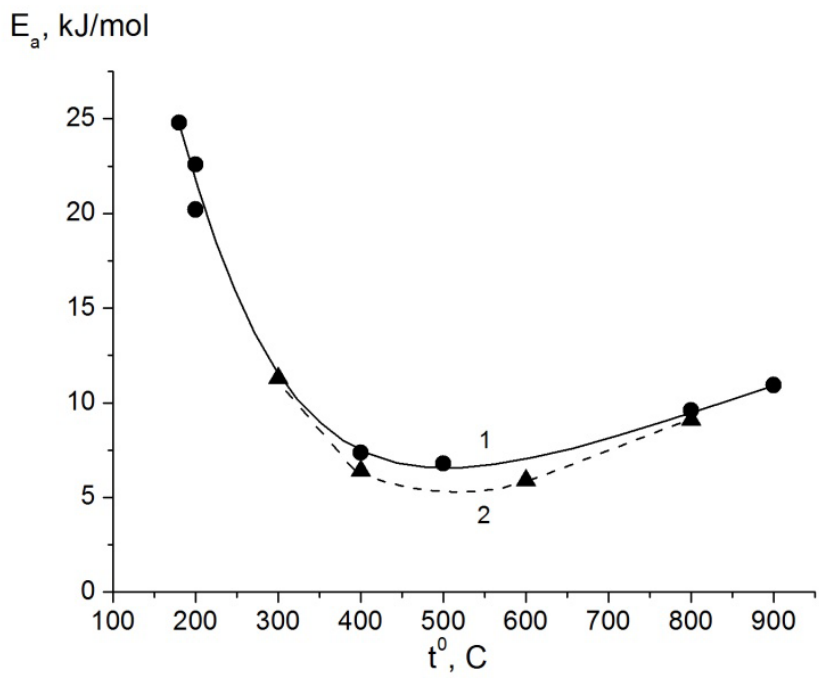

Figure 7. Dependence of apparent activation energies of photoinduced oxidation of formaldehyde to formic acid on calcination temperatures of titanium dioxides (1) and 10\% rutile-anatase composite (2). (All samples of titanium dioxide and composite were subjected to calcinations for 3 hours).

\section{Conclusion}

Thus, the way of synthesis of the rutile-anatase composite catalyst is developed by deposition of the titanium(IV) oxyhydroxide on a surface of rutile particles with the subsequent it dehydration and calcination for formation of an anatase crystal phase. To obtain composites with reproductive physico-chemical properties during synthesizing special attention was paid to control of its basic parameters. Concentrations of metal ions, hydrogen and rutile particles, the degree of mother liquor supersaturation, the tome of hydroxide ageing, temperature and time of composite drying and calcination were considered as above mentioned parameters.

Investigations of formaldehyde photoinduced oxidation on modificated by anatase particles rutile has shown its great activity which can be evidence that there exists synergism of photoinduced catalytic activity of two coupled crystal structures of titanium dioxide. The modification of rutile surface by particles of another semiconductor can be used as a perspective way of the designing of new improved photocatalytic systems.

\section{REFERENCES}

[1] M.R. Hoffman, S.T. Martin, W. Choi, D.W. Bahnemann. Environmental applications of semiconductor photocatalysis, Chemical Reviews, Vol. 95, 69-96, 1995.

[2] O. Carp, C.L. Huisman, A. Reller. Photoinduced reactivity of titanium dioxide, Progress in Solid State Chemistry, Vol. 32, 33-177, 2004.

[3] K. Lu, J. Yu, K. Deng, X. Li, M. Li. Effect of phase 
structures on the formation rate of hydroxyl radicals on the surface of TiO2,J. Phys. Chem. Solids, Vol. 71, No 4, 519-522, 2010

[4] J. Xu, W. Wang, S. Sun, L. Wang. Enhancing visible-light-induced photocatalytic activity by coupling with wide-band-gap semiconductor: A case study on Bi2WO6/TiO2, Applied Catalysis B: Environmental, Vol. 111, 126-132, 2012.

[5] A. Nikolenko, B. Melnykov. Photocatalytic Oxidation of Formaldehyde Vapour Using Amorphous Titanium Dioxide, Chem. \& Chem. Techn., Vol. 4, 311-315, 2010.

[6] M. Graetzel, F. Rotzinger P. Raman spectroscopic evidence for the existence of titanyl $\left(\mathrm{TiO}^{2+}\right)$ in acidic aqueous solutions, Inorg. Chem., Vol. 24, 2320-2321, 1985.

[7] H. Einage, Y. Komatsu. Hydrolytic precipitation reaction of titanium(IV) from $(\mathrm{Na}, \mathrm{H}) \mathrm{Cl}$ aqueous solution, J. Inorg. Nucl. Chem, Vol. 43, 2443-2448, 1981.

[8] A. Tsevis, N. Spanos, P.G. Koutsoukos, A.J. Linde, J. Lyklema. Preparation and characterization of anatase powders , J. Chem. Soc., Faraday Trans., Vol. 94, 295-300, 1998.

[9] J. Subrt, V. Stengel, S. Bakardjieva, L. Szatmary. Synthesis of spherical metal oxide particles using homogeneous precipitation of aqueous solutions of metal sulfates with urea, Powder Technology, Vol. 169, 33-40, 2006.

[10] S. Yamabi, H. Imai. Synthesis of rutile and anatase fims with high surface areas in aqueous solutions containing urea, Thin Solid Films, Vol. 434, 86-93, 2003.

[11] В.Г. Верещак, М.В. Ніколенко, А.М. Калашникова. Одержання малоагрегованих оксидних порошків, ВXXТ,
2013, Vol. 3, 53-58.

[12] B. Huang, S. Saka. Photocatalytic activity of $\mathrm{TiO} 2$ crystallite - activated carbon composites prepared in supercritical isopropanol for the decomposition of formaldehyde, J.Wood Sci., Vol. 49, 79-85, 2003.

[13] X. Xiao, D. Liao, H. Zhang. Synthesis of TiO2 nano-particles and their photocatalytic activity for formaldehyde and methyl orange degradation, Front. Chem. Eng. China, Vol.1(2), 178-183, 2007.

[14] Y. Qing, G. Fen, X. Ying, X. Cai-jun, G. Bao-wen. Фотокаталитическая деструкция формальдегида на пористой пленке из TiO2, Huanjing kexue, Vol. 26, No 4, 35-39, 2005.

[15] Y. Jian-Jun, L. Dong-Xu, L. Qing-Lin, Z. Zhi-Jun, W. Han-Qing. Mechnism of Photocatalytic Oxidation of Formaldehyde, J. Acta Phys. Chim. Sin., Vol. 17, No 3, 278-281, 2001.

[16] S. Song, D. Jianjun, B. Jun, G. Chen, Q. Zeming. Photocatalytic Oxidation of Gaseous Formaldehyde on TiO2: An In Situ DRIFTS Study, Catal. Lett., Vol. 137, 239-246, 2010.

[17] C. Zhang, H. He, K. Tanaka. Catalytic performance and mechanism of a $\mathrm{Pt} / \mathrm{TiO}_{2}$ catalyst for the oxidation of formaldehyde at room temperature Appl. Catal. B, Environ., Vol. 65, 3743, 2006.

[18] J.L. Shie, C.H. Lee, C.S. Chiou, C.T. Chang, C.C. Chang, C.Y. Chang. Photodegradation kinetics of formaldehyde using light sources of UVA, UVC and UVLED in the presence of composed silver titanium oxide photocatalyst, J. Hazard. Mater., Vol. 155, No1-2, 164-172, 2008. 